\title{
Green Femtocell Networks through Joint Power Control and 3-Dimensional Pattern-Dependent Beamforming
}

\author{
Narges NOORI \\ Dept. of Communication Technology, Iran Telecommunication Research Center, Tehran, Iran \\ nnoori@itrc.ac.ir
}

Submitted April 26, 2017 / Accepted June 12, 2017

\begin{abstract}
Femtocell technologies are used to extend radio coverage into buildings and provide wireless broadband access in residential, office or indoor hotspot environments. However, due to the radio spectrum scarcity, the femtocells reuse the same licensed spectrum bands of the macrocells. Thus, interference management is one of the main challenges under such co-channel deployment of the femtocells. In this paper, we propose a new joint power control and 3-dimensional (3D) pattern-dependent beamforming algorithm to minimize total power consumption of the femtocells deployed in a macrocellular environment. This algorithm can help us achieve the goal of green communication and significantly reduce power consumption of the overall radio access networks. The algorithm is based on an iterative scheme for a downlink case where all femtocell access points (FAPs) and macro base stations (BSs) are equipped with multiple antennas. By considering radiation pattern of all antennas, the scheme tries to find the optimal $3 D$ beamforming weights and transmission power of all FAPs and BSs while satisfying minimum signal-to-interference-plus-noise ratio (SINR) requirements of both macrocell and femtocell networks. Simulations are conducted in various scenarios to evaluate performance of the proposed algorithm.
\end{abstract}

\section{Keywords}

Femtocells, power control, 3D beamforming, green communication

\section{Introduction}

With the advent of new hand-held devices and smart phones, data intensive applications such as online music and video streaming, video conferencing and network gaming have occupied more users' attention. Recent studies show that more than $50 \%$ of all voice calls and $70 \%$ of data traffic are generated in indoor environments [1]. However, poor radio coverage inside buildings caused by wall penetration losses makes it difficult for indoor cellular users to receive high-quality services from a macro base station (BS). On the other hand, increasing the BS transmit power does not solve the problem as it leads to more co-channel interference into neighboring cells. Furthermore, it is very costly to have a large number of macrocellular BSs to give better indoor coverage and meet the needs of high capacity networks.

Femtocells are small-coverage, low cost and plugand-play indoor access points designed to solve in-building coverage and capacity challenges and accordingly support mobile broadband services [2], [3]. These access points connect indoor users to the cellular core network via their broadband internet access connections [1], [4]. The users must pay additional fees to the cellular operator as well as the cost for the internet connection to route the femtocell traffic. When cellular users are located inside buildings, they are often connected to the nearby femtocell access points (FAPs) rather than a distant BS to receive high quality voice, data and multimedia services with much lower power consumption. However, due to the scarcity of the spectrum, femtocells normally need to share the spectrum resources with the macrocell networks. The femtocell users can access the frequency bands of the macrocells as long as not causing harmful interference to those networks [5], [6]. In the coexisting macrocell-femtocell heterogeneous networks, interference exists between the femtocells and macrocell as well as between neighboring femtocells, especially in dense femtocell deployment scenarios [7]. Furthermore, huge deployment of the femtocells will ultimately make energy efficiency one of the major challenges of these networks [8].

As mentioned above, one of the main challenges faced by femtocell networks is interference management. Thus, mitigating cross-tier (femto-to-macro and macro-tofemto) and co-tier (femto-to-femto) interference, while maintaining quality of service (QoS) or spectrum efficiency, has attracted many research interests [5], [9-13]. Multiple antenna techniques can be applied as an effective way to improve interference mitigation [14-16]. On the other hand, energy efficient deployment of the femtocell network has been investigated in [17-19] via power control.

The combined use of transmit power control and beamforming for femtocells was considered in [16] by investigating the effect of channel uncertainty. Accord- 
ingly, the transmit power level and beam weights are determined to provide desired signal-to-interference-plusnoise ratio (SINR) for femto users (FUs) while minimizing interference among the serving FUs and adjacent macro users (MUs). However, this work studied interference mitigation and did not address energy efficiency of the deployed network. In [19], two distributed downlink power allocation schemes were proposed to maximize energy efficiency in the femtocells while satisfying minimum SINR requirements of all MUs and FUs.

Recently, 3-dimensional (3D) beamforming algorithms are proposed for interference mitigation in cellular networks. These algorithms provide significant improvements against 2-dimensional traditional beamforming techniques [20-22]. In 3D beamforming, the enhanced beamforming algorithms are applied to obtain beamforming weights according to both users' horizontal and vertical positions. The aim of this paper is to propose a new beamforming technique for interference management and energy efficient deployment of the macrocell-femtocell heterogeneous networks. Different from most of the research in the current literature on the joint power control and beamforming algorithms for cellular interference management, which are based on the 2-dimensional beamforming, we propose a new 3D pattern-dependent beamforming scheme. Since a precise $3 \mathrm{D}$ beamforming is correlated to the radiation pattern of the array elements, the proposed joint power control and 3D beamforming scheme is formulated by considering radiation pattern of the antennas to minimize total power consumption of the whole network and achieve environment-friendly green communication. This scheme attempts to find power values and beamforming weights of the FAPs and macrocell BS in the downlink scenario by satisfying the SINR requirements of both macrocell and femtocell networks. The well-known WINNER II channel model [23] is applied for path loss estimation in the 3D propagation environment of the considered system. The performance of this algorithm and the effects of the antenna pattern in terms of the consumed power will be shown through numerical simulations.

The organization of this paper is as follows: Section 2 provides a detailed system model. In Sec. 3, a 3D patterndependent joint power control and beamforming scheme is introduced to minimize the total transmission power of the whole system. Numerical simulation results are presented in Sec. 4. Finally, Section 5 concludes the paper.

\section{System Model}

According to Fig. 1, we consider downlink of a heterogeneous wireless system where $K$ office/residential femtocells share a frequency band with an urban macrocell network with $L_{M}$ MUs. The BS is equipped with a uniform planar antenna array of $N_{M}^{c}$ similar antennas in each row (each row is a uniform linear array) and a total number of $N^{r}{ }_{M}$ similar rows in the vertical dimension (each column is also a uniform linear array). Similarly, each FAP is equipped with a uniform planar antenna array with $N_{F}^{c}$ similar antennas in each row and a total number of $N_{F}^{r}$ similar rows in the vertical dimension. There are $L_{F}$ FUs in each femtocell. All MUs and FUs have a single omnidirectional antenna. The FAPs can access the core network of the mobile operator via a broadband internet backhaul.

In this system, we focus on two interference scenarios: macro-to-femto interference (interference from the BS to the FUs) and femto-to-macro interference (interference from the FAP to the MUs). We also assume that the interference from a femtocell to the other femtocells is negligible. The symbol $l_{M}\left(l_{M}=1,2, \ldots, L_{M}\right)$ is used to denote the index of a MU, while $l_{F}\left(l_{F}=1,2, \ldots, L_{F}\right)$ points to that of

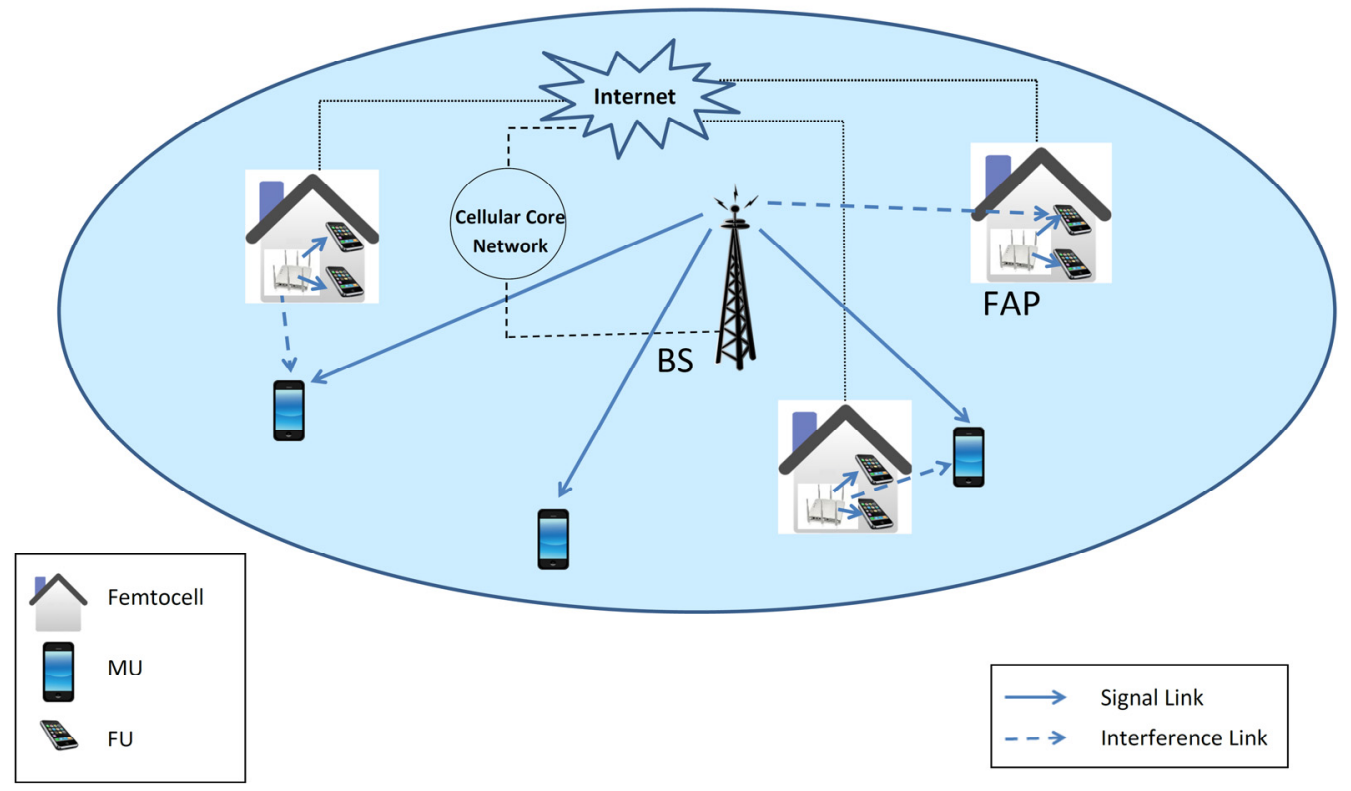

Fig. 1. Model for downlink of a macrocell-femtocell heterogeneous system. 
a FU in each femtocell. The channel gains from the BS to the $l_{M}$-th MU and to the $l_{F}$-th FU of the $k$-th femtocell are defined by $g_{B l_{M}}$ and $g_{B l_{F}}^{k}$, respectively $(k=1,2, \ldots, K)$. Similarly, the channel gains from the $k$-th FAP to the $l_{M}$-th MU and to the $l_{F}$-th FU of the corresponding femtocell are denoted by $g_{F l_{M}}^{k}$ and $\tilde{g}_{F l_{F}}^{k}$, respectively. A 3D channel model is required to obtain path loss gains in this system. The WINNER II channel models are provided for link level and system level simulations of local area, metropolitan area, and wide area wireless communication systems operating in $2-6 \mathrm{GHz}$ frequency range [23]. The models support multi-antenna technologies, polarization, multiuser, multi-cell, and multi-hop networks. By applying the WINNER II channel model, the path loss can be written as [23]:

$$
P L=A \log _{10} d+B+C \log _{10}\left(\frac{f_{c}}{5.0}\right)+X
$$

where $d$ is the distance between the transmitter and receiver in $[\mathrm{m}], f_{c}$ is the system frequency in $[\mathrm{GHz}]$, the fitting parameter $A$ includes the path loss exponent, $B$ is the intercept, $C$ describes the path loss frequency dependence and $X$ is an optional environment-specific term. This model can be applied for frequencies in the range of 2 to $6 \mathrm{GHz}$ with different antenna heights. The corresponding parameters of (1) for all propagation scenarios of in-building, urban, indoor to outdoor and outdoor to indoor within our considered system can be extracted from [23].

Assuming that the signal is transmitted for each MU through beamforming, the output of the array elements at the BS are weighted and added by a 3D beamformer. This can steer a main beam toward the target user and adjust the nulls to reject the interference in the direction of the others. Although, in precise interference cancelation, the radiation pattern of the array elements must be taken into account, but the issue has not been considered in general beamforming schemes. In this paper, the channel responses are formulated by considering radiation pattern of the antennas in the BS and all FAPs. If the radiation pattern of the BS elements is represented by $f_{B}(\theta, \varphi)$, the channel responses between the BS and the $l_{M}$-th MU and between the BS and the $l_{\mathrm{F}}$-th FU of the $k$-th femtocell can be denoted by $\mathbf{h}_{B l_{M}(}\left(\theta_{B l_{M}}, \varphi_{\left.B l_{M}\right)}\right.$ and $\mathbf{h}_{B l_{F}}^{k}\left(\theta_{B l_{F}}^{k}, \varphi_{B l_{F}}^{k}\right.$, respectively:

$$
\begin{gathered}
\mathbf{h}_{B l_{M}}\left(\theta_{B l_{M}}, \varphi_{B l_{M}}\right)=\sqrt{g_{B l_{M}}} f_{B}\left(\theta_{B l_{M}}, \varphi_{B l_{M}}\right) \mathbf{v}\left(\theta_{B l_{M}}, \varphi_{B l_{M}}\right) \\
=\sqrt{g_{B l_{M}}} f_{B}\left(\theta_{B l_{M}}, \varphi_{B l_{M}}\right) \mathbf{v}_{v}\left(\theta_{B l_{M}}\right) \otimes \mathbf{v}_{h}\left(\theta_{B l_{M}}, \varphi_{B l_{M}}\right), \\
\forall l_{M} \in\left[1, L_{M}\right] \\
\mathbf{h}_{B l_{F}}^{k}\left(\theta_{B l_{F}}^{k}, \varphi_{B l_{F}}^{k}\right)=\sqrt{g_{B l_{F}}} f_{B}\left(\theta_{B l_{F}}^{k}, \varphi_{B l_{F}}^{k}\right) \mathbf{v}\left(\theta_{B l_{F}}^{k}, \varphi_{B l_{F}}^{k}\right) \\
=\sqrt{g_{B l_{F}}} f_{B}\left(\theta_{B l_{F}}^{k}, \varphi_{B l_{F}}^{k}\right) \mathbf{v}_{v}\left(\theta_{B l_{F}}^{k}\right) \otimes \mathbf{v}_{h}\left(\theta_{B l_{F}}^{k}, \varphi_{B l_{F}}^{k}\right), \\
\forall l_{F} \in\left[1, L_{F}\right]
\end{gathered}
$$

with

$$
\begin{gathered}
\mathbf{v}_{v}(\theta)=\left[1, \mathrm{e}^{-\mathrm{j} 2 \pi \frac{d_{v}}{\lambda} \sin \theta}, \ldots, \mathrm{e}^{-\mathrm{j} 2 \pi\left(N_{M}^{r}-1\right) \frac{d_{v}}{\lambda} \sin \theta}\right]^{\mathrm{T}}, \\
\mathbf{v}_{h}(\theta, \varphi)=\left[1, \mathrm{e}^{-\mathrm{j} 2 \pi \frac{d_{h}}{\lambda} \cos \theta \sin \varphi}, \ldots, \mathrm{e}^{-\mathrm{j} 2 \pi\left(N_{M}^{c}-1\right) \frac{d_{h}}{\lambda} \cos \theta \sin \varphi}\right]^{\mathrm{T}}
\end{gathered}
$$

where $\otimes$ denotes the Kronecker product, $(\cdot)^{\mathrm{T}}$ defines the transpose operation, $\theta_{B l_{M}}$ and $\varphi_{B l_{M}}$ are the angles of departure from the BS to the $l_{M}$-th MU in the vertical and horizontal directions, respectively. Similarly, $\theta_{B l_{F}}^{k}$ and $\varphi_{B l_{F}}^{k}$ are the angles of departure from the BS to the $l_{F}$-th FU of the $k$-th femtocell in the vertical and horizontal directions, respectively. Furthermore, $d_{v}$ and $d_{h}$ are the distances between two adjacent array antenna elements in a column and a row, respectively. Correspondingly, if the radiation pattern of the $k$-th FAP elements is represented by $f_{F}^{k}(\theta, \varphi)$, the channel responses between the $k$-th FAP and the $l_{M}$-th MU and between the $k$-th FAP and the $l_{F}$-th FU of the corresponding femtocell can be represented by $\mathbf{h}_{F l_{M}}^{k}\left(\theta_{F l_{M}}^{k}, \varphi_{\left.F l_{M}\right)}^{k}\right.$ and $\tilde{\mathbf{h}}_{F l_{F}}^{k}\left(\theta_{F l_{F}}^{k}, \varphi_{F l_{F}}^{k}\right.$, respectively:

$$
\begin{gathered}
\mathbf{h}_{F l_{M}}^{k}\left(\theta_{F l_{M}}^{k}, \varphi_{F l_{M}}^{k}\right)=\sqrt{g_{F l_{M}}^{k}} f_{F}^{k}\left(\theta_{F l_{M}}^{k}, \varphi_{F l_{M}}^{k}\right) \mathbf{v}\left(\theta_{F l_{M}}^{k}, \varphi_{F l_{M}}^{k}\right) \\
=\sqrt{g_{F l_{M}}^{k}} f_{F}^{k}\left(\theta_{F l_{M}}^{k}, \varphi_{F l_{M}}^{k}\right) \mathbf{v}_{v}\left(\theta_{F l_{M}}^{k}\right) \otimes \mathbf{v}_{h}\left(\theta_{F l_{M}}^{k}, \varphi_{F l_{M}}^{k}\right), \\
\forall l_{M} \in\left[1, L_{M}\right] \\
\tilde{\mathbf{h}}_{F l_{F}}^{k}\left(\theta_{F l_{F}}^{k}, \varphi_{F l_{F}}^{k}\right)=\sqrt{\tilde{g}_{F l_{F}}^{k}} f_{F}^{k}\left(\theta_{F l_{F}}^{k}, \varphi_{F l_{F}}^{k}\right) \mathbf{v}\left(\theta_{F l_{F}}^{k}, \varphi_{F l_{F}}^{k}\right) \\
=\sqrt{\tilde{g}_{F l_{F}}^{k}} f_{F}^{k}\left(\theta_{F l_{F}}^{k}, \varphi_{F l_{F}}^{k}\right) \mathbf{v}_{v}\left(\theta_{F l_{F}}^{k}\right) \otimes \mathbf{v}_{h}\left(\theta_{F l_{F}}^{k}, \varphi_{F l_{F}}^{k}\right), \\
\forall l_{F} \in\left[1, L_{F}\right]
\end{gathered}
$$

where $\theta_{F l_{M}}^{k}$ and $\varphi_{F l_{M}}^{k}$ are the angles of departure from the $k$ th FAP to the $l_{M}$-th MU in the vertical and horizontal directions, respectively. Similarly, $\theta_{F l_{F}}^{k}$ and $\varphi_{F l_{F}}^{k}$ are the angles of departure from the $k$-th FAP to the $l_{F}$-th FU of the corresponding femtocell in the vertical and horizontal directions, respectively.

Let us denote $\mathbf{w}_{l_{M}}$ as the $N^{r}{ }_{M} N^{c}{ }_{M}$-component complex weight vector for the $l_{M}$-th $\mathrm{MU}$, and $\mathbf{w}_{l_{F}}^{k}$ as the $N_{F}^{r} N_{F^{-}}^{c}$ component complex weight vector for the $l_{F}$-th FU of the $k$-th femtocell. Then, the received signal at the $l_{M}$-th $\mathrm{MU}$ can be represented by:

$$
\begin{aligned}
y_{l_{M}=} & \sqrt{p_{l_{M}}} \mathbf{w}_{l_{M}}^{\mathrm{H}} \mathbf{h}_{B l_{M}} s_{l_{M}}+\sum_{\substack{i=1 \\
i \neq l_{M}}}^{L_{M}} \sqrt{p_{i}} \mathbf{w}_{i}^{\mathrm{H}} \mathbf{h}_{B l_{M}} s_{i}+ \\
& \sum_{k=1}^{K} \sum_{j=1}^{L_{F}} \sqrt{p_{j}^{k}} \mathbf{w}_{j}^{k \mathrm{H}} \mathbf{h}_{F l_{M}}^{k} s_{j}^{k}+n_{l_{M}}, \forall k \in[1, K], l_{M} \in\left[1, L_{M}\right]
\end{aligned}
$$

where $p_{i}$ and $p_{j}^{k}$ are the transmission power values intended for the $i$-th MU and the $j$-th FU of the $k$-th femtocell, respectively, $s_{i}$ and $s_{j}^{k}$ are the signals transmitted to the $i$-th MU and the $j$-th FU, respectively, $n_{l_{M}}$ denotes the zeromean additive white Gaussian noise (AWGN) of the $l_{M^{-}}$th $\mathrm{MU}$, and $(\cdot)^{\mathrm{H}}$ defines the Hermitian transpose operation. 
Also, the received signal at the $l_{F}$-th FU of the $k$-th femtocell can be written as:

$$
\begin{aligned}
y_{l_{F}}^{k}= & \sqrt{p_{l_{F}}^{k}} \mathbf{w}_{l_{F}}^{k \mathrm{H}} \tilde{\mathbf{h}}_{F l_{F}}^{k} \tilde{s}_{m_{F}}^{k}+\sum_{i=1}^{L_{M}} \sqrt{p_{i}} \mathbf{w}_{i}^{\mathrm{H}} \mathbf{h}_{B l_{F}}^{k} s_{i}+ \\
& \sum_{\substack{j=1 \\
j \neq l_{F}}}^{L_{F}} \sqrt{p_{j}^{k}} \mathbf{w}_{j}^{k \mathrm{H}} \tilde{\mathbf{h}}_{F_{F}}^{k} \tilde{s}_{j}^{k}+n_{l_{F}}^{k}, \quad \forall k \in[1, K], l_{F} \in\left[1, L_{F}\right]
\end{aligned}
$$

where $n_{l F}^{k}$ represents the zero-mean AWGN of the $l_{F}$-th FU of the $k$-th femtocell. The first terms in both (8) and (9) are the desired signals, while the other terms are related to the interference and noise.

Assuming that the message signals are uncorrelated and have zero-mean, the SINR values at the $l_{M}$-th MU and the $l_{F}$-th FU of the $k$-th femtocell are given respectively by:

$$
\gamma_{l_{M}}=\frac{p_{l_{M}}\left|\mathbf{w}_{l_{M}}^{\mathrm{H}} \mathbf{h}_{B l_{M}}\right|^{2}}{\sum_{\substack{i=1 \\ i \neq l_{M}}}^{L_{M}} p_{i}\left|\mathbf{w}_{i}^{\mathrm{H}} \mathbf{h}_{B l_{M}}\right|^{2}+\sum_{k=1}^{K} \sum_{j=1}^{L_{F}} p_{j}^{k}\left|\mathbf{w}_{j}^{k \mathrm{H}} \mathbf{h}_{F l_{M}}^{k}\right|^{2}+\sigma_{l_{M}}^{2}}, \quad \forall l_{M} \in\left[1, L_{M}\right],
$$$$
\gamma_{l_{F}}^{k}=\frac{p_{l_{F}}^{k}\left|\mathbf{w}_{l_{F}}^{k \mathrm{H}} \tilde{\mathbf{h}}_{F_{l_{F}}}^{k}\right|^{2}}{\sum_{i=1}^{L_{M}} p_{i}\left|\mathbf{w}_{i}^{\mathrm{H}} \mathbf{h}_{B l_{F}}^{k}\right|^{2}+\sum_{\substack{j=1 \\ j \neq l_{F}}}^{L_{F}} p_{j}^{k}\left|\mathbf{w}_{j}^{k \mathrm{H}} \tilde{\mathbf{h}}_{F_{l_{F}}}^{k}\right|^{2}+\sigma_{l_{F}, k}^{2}}, \quad \forall k \in[1, K], l_{F} \in\left[1, L_{F}\right]
$$

where $\sigma_{l_{M}}^{2}$ and $\sigma_{l F, k}^{2}$ are the receiver noise variance of the $l_{M}$-th MU and the $l_{F}$-th FU of the $k$-th femtocell, respectively.

\section{Joint Power Control and Beamforming}

In our joint power control and beamforming problem, the objective is to find the optimal weight vector and power allocations such that the SINR threshold is achieved by all system links, while total transmission power of the whole system is minimized. This reduces power consumption of the radio access networks and helps achieve the goal of environment-friendly green communications. To this end, the optimization problem is defined by:

$$
\begin{aligned}
& \min _{\left\{w_{i}, w_{j}^{k}\right\},\left\{p_{i}, p_{j}^{k}\right\}}\left(\sum_{i=1}^{L_{M}} p_{i}+\sum_{k=1}^{K} \sum_{j=1}^{L_{F}} p_{j}^{k}\right), \\
& \text { subject to }: \gamma_{l_{M}} \leq \tilde{\gamma}_{l_{M}} \text { and } \gamma_{l_{F}}^{k} \leq \tilde{\gamma}_{l_{F}}^{k}, \\
& \forall l_{M}, k, l_{F}
\end{aligned}
$$

where $\tilde{\gamma}_{l_{M}}$ and $\tilde{\gamma}_{l_{F}}^{k}$ denote the minimum required SINR of the $l_{M}$-th MU and the $l_{F}$-th FU of the $k$-th femtocell, respectively. form as

The constraints of (12) can be presented in the matrix

$$
\left(\mathbf{I}_{Q}-\mathbf{D G}\right) \mathbf{p} \geq \mathbf{u}
$$

where $\mathbf{I}_{Q}$ is an identity matrix of size $Q=L_{M}+K L_{F}$, the diagonal matrix $\mathbf{D}$ is defined by:

$$
\begin{array}{r}
\mathbf{D}=\operatorname{diag}\left\{\frac{\tilde{\gamma}_{1}}{\left|\mathbf{w}_{1}^{\mathrm{H}} \mathbf{h}_{B 1}\right|^{2}}, \ldots, \frac{\tilde{\gamma}_{L_{M}}}{\left|\mathbf{w}_{L_{M}}^{\mathrm{H}} \mathbf{h}_{B L_{M}}\right|^{2}}, \frac{\tilde{\gamma}_{1}^{1}}{\left|\mathbf{w}_{1}^{1 \mathrm{H}} \tilde{\mathbf{h}}_{F 1}^{1}\right|^{2}}, \ldots,\right. \\
\left.\frac{\tilde{\gamma}_{L_{F}}^{1}}{\left|\mathbf{w}_{L_{F}}^{1 \mathrm{H}} \tilde{\mathbf{h}}_{F L_{F}}^{1}\right|^{2}}, \ldots, \frac{\tilde{\gamma}_{1}^{K}}{\left|\mathbf{w}_{1}^{K \mathrm{H}} \tilde{\mathbf{h}}_{F 1}^{K}\right|^{2}}, \ldots, \frac{\tilde{\gamma}_{L_{F}}^{K}}{\left|\mathbf{w}_{L_{F}}^{K \mathrm{H}} \tilde{\mathbf{h}}_{F L_{F}}^{K}\right|^{2}}\right\},
\end{array}
$$

and the $\left(L_{M}+K L_{F}\right) \times\left(L_{M}+K L_{F}\right)$ matrix $\mathbf{G}$ is given by:

$$
\mathbf{G}=\left[\begin{array}{ll}
\mathbf{G}_{1} & \mathbf{G}_{2} \\
\mathbf{G}_{3} & \mathbf{G}_{4}
\end{array}\right]
$$

Here, $\mathbf{G}_{1}, \mathbf{G}_{2}, \mathbf{G}_{3}$, and $\mathbf{G}_{4}$ are $L_{M} \times L_{M}, L_{M} \times K L_{F}, K L_{F} \times L_{M}$ and $K L_{F} \times K L_{F}$ submatrices, respectively. If we represent the element at the $m$-th row and $n$-th column of the matrix $\mathbf{G}_{i}$ by $\left[G_{i}\right]_{m, n}$, then we have:

$$
\begin{gathered}
{\left[G_{1}\right]_{m, n}=\left\{\begin{array}{cc}
0 & \text { for } m=n \\
\left|\mathbf{w}_{n}^{\mathrm{H}} \mathbf{h}_{B m}\right|^{2} & \text { for } m \neq n
\end{array},\right.} \\
{\left[G_{2}\right]_{m, n}=\left|\left(\mathbf{w}_{n-L_{F} \cdot \tilde{n}}^{\tilde{n}+1}\right)^{\mathrm{H}} \mathbf{h}_{F m}^{\tilde{n}+1}\right|^{2},} \\
{\left[G_{3}\right]_{m, n}=\left|\mathbf{w}_{n}^{\mathrm{H}} \mathbf{h}_{B\left(m-L_{F} \cdot \tilde{m}\right)}^{\tilde{n}+1}\right|^{2},}
\end{gathered}
$$

$\left[G_{4}\right]_{m, n}=\left\{\begin{array}{cc}\left|\left(\mathbf{w}_{n-L_{F} \cdot \tilde{n}}^{\tilde{m}+1}\right)^{\mathrm{H}} \tilde{\mathbf{h}}_{F\left(m-L_{F} \cdot \tilde{m}\right)}^{\tilde{m}+1}\right| & \text { for } L_{F} \cdot \tilde{m}+1 \leq n< \\ 0 & L_{F} \cdot(\tilde{m}+1), m \neq n \\ 0 & \text { otherwise },\end{array}\right.$

where $\quad \tilde{m}=(m-1) \operatorname{div} L_{F} \quad$ and $\quad \tilde{n}=(n-1) \operatorname{div} L_{F}$. The $\left(L_{M}+K L_{F}\right) \times 1$ vectors $\mathbf{p}$ and $\mathbf{u}$ are given, respectively by:

$$
\begin{gathered}
\mathbf{p}=\left[p_{1}, \ldots, p_{L_{M}}, p_{1}^{1}, \ldots, p_{L_{F}}^{1}, \ldots, p_{1}^{K}, \ldots, p_{L_{F}}^{K}\right]^{\mathrm{H}}, \\
\mathbf{u}=\left[\frac{\tilde{\gamma}_{1} \sigma_{1}^{2}}{\left|\mathbf{w}_{1}^{\mathrm{H}} \mathbf{h}_{B 1}\right|^{2}}, \ldots, \frac{\tilde{\gamma}_{L_{M}} \sigma_{L_{M}}^{2}}{\left|\mathbf{w}_{L_{M}}^{\mathrm{H}} \mathbf{h}_{B L_{M}}\right|^{2}}, \frac{\tilde{\gamma}_{1}^{1} \sigma_{1,1}^{2}}{\left|\mathbf{w}_{1}^{1 \mathrm{H}} \mathbf{h}_{F 1}^{1}\right|^{2}}, \ldots, \frac{\tilde{\gamma}_{1}^{1} \sigma_{1,1}^{2}}{\left|\mathbf{w}_{L_{F}}^{1 \mathrm{H}} \tilde{\mathbf{h}}_{F L_{F}}^{1}\right|^{2}},\right. \\
\left.\ldots, \frac{\tilde{\gamma}_{1}^{K} \sigma_{1, K}^{2}}{\left|\mathbf{w}_{1}^{K \mathrm{H}} \mathbf{h}_{F 1}^{K}\right|^{2}}, \ldots, \frac{\tilde{\gamma}_{L_{F}}^{K} \sigma_{L_{F}, K}^{2}}{\left|\mathbf{w}_{L_{F}}^{K \mathrm{H}} \tilde{\mathbf{h}}_{F L_{F}}^{K}\right|^{2}}\right]^{\mathrm{T}} .
\end{gathered}
$$

Then, the vector

$$
\mathbf{p}_{w}=\left(\mathbf{I}_{Q}-\mathbf{D G}\right)^{-1} \mathbf{u}
$$

minimizes the objective function in (12) for the fixed weight vector set $\mathbf{w}$.

However, direct optimization of the downlink scenario in (12) is complicated. For this reason, we use the duality concept between uplink and downlink multiuser channels [24-26] and solve the downlink problem by converting it into virtual uplink one. In our system, the virtual uplink algorithm can be obtained by using the receive 
beamforming vector in the BS and FAPs in the reciprocal transmission to the same user. Since the optimal uplink receive beamforming vector is also the optimal downlink transmission vector, a virtual uplink network is constructed whose channel responses are the same as that of the downlink. In such a scenario, we can write the SINR at the beamformer output corresponding to the $l_{M}$-th $\mathrm{MU}$ and the $l_{F}$-th FU of the $k$-th femtocell, respectively, as:

$$
\begin{aligned}
& \gamma_{l_{M}}^{\prime}=\frac{\tilde{p}_{l_{M}}\left|\mathbf{w}_{l_{M}}^{\mathrm{H}} \mathbf{h}_{B l_{M}}\right|^{2}}{\sum_{\substack{i=1 \\
i \neq l_{M}}}^{L_{M}} \tilde{p}_{i}\left|\mathbf{w}_{l_{M}}^{\mathrm{H}} \mathbf{h}_{B i}\right|^{2}+\sum_{k=1}^{K} \sum_{j=1}^{L_{F}} \tilde{p}_{j}^{k}\left|\mathbf{w}_{l_{M}}^{\mathrm{H} \mathrm{H}} \mathbf{h}_{B j}^{k}\right|^{2}+\sigma_{l_{M}}^{2}\left\|\mathbf{w}_{l_{M}}^{\mathrm{H}}\right\|^{2}}, \\
& \gamma_{l_{F}}^{\prime k}=\frac{\tilde{p}_{l_{F}}^{k}\left|\mathbf{w}_{l_{F}}^{k \mathrm{H}} \tilde{\mathbf{h}}_{F l_{F}}^{k}\right|^{2}}{\sum_{i=1}^{L_{M}} \tilde{p}_{i}\left|\mathbf{w}_{l_{F}}^{k \mathrm{H}} \mathbf{h}_{B l_{F}}^{k}\right|^{2}+\sum_{\substack{j=1 \\
j \neq l_{F}}}^{L_{F}} \tilde{p}_{j}^{k}\left|\mathbf{w}_{l_{F}}^{k \mathrm{H}} \tilde{\mathbf{h}}_{F j}^{k}\right|^{2}+\sigma_{l_{F}, k}^{2}\left\|\mathbf{w}_{l_{F}}^{k \mathrm{H}}\right\|^{2}}
\end{aligned}
$$

where $\tilde{p}_{l_{M}}$ and $\tilde{p}_{l_{F}}^{k}$ are the virtual uplink transmission power values of the $l_{M}$-th $\mathrm{MU}$ and the $l_{F}$-th FU in the $k$-th femtocell, respectively. Furthermore, $\sigma_{l_{M}}^{2}$ and $\sigma_{l_{F, k}}^{2}$ are receiver noise variances for the $l_{M}$-th MU and for the $l_{F}$-th FU of the $k$-th femtocell, respectively. In (23) and (24), the SINR values are coupled only by the transmission power. By exploiting this fact, we convert the downlink framework into the virtual uplink one and provide an iterative algorithm to find the weight vectors and solve the optimization problem defined in (12). In the virtual uplink scenario, Equation (22) can be rewritten as:

$$
\tilde{\mathbf{p}}=\left(\mathbf{I}_{Q}-\mathbf{D} \mathbf{G}^{\mathrm{T}}\right)^{-1} \tilde{\mathbf{u}}
$$

where

$$
\begin{aligned}
\mathbf{p}= & {\left[\tilde{p}_{1}, \ldots, \tilde{p}_{L_{M}}, \tilde{p}_{1}^{1}, \ldots, \tilde{p}_{L_{F}}^{1}, \ldots, \tilde{p}_{1}^{K}, \ldots, \tilde{p}_{L_{F}}^{K}\right]^{\mathrm{H}}, } \\
\tilde{\mathbf{u}}= & {\left[\frac{\tilde{\gamma}_{1} \tilde{\sigma}_{1}^{2}\left\|\mathbf{w}_{1}^{\mathrm{H}}\right\|^{2}}{\left|\mathbf{w}_{1}^{\mathrm{H}} \mathbf{h}_{B 1}\right|^{2}}, \ldots, \frac{\tilde{\gamma}_{L_{M}} \tilde{\sigma}_{L_{M}}^{2} \|\left.\mathbf{w}_{L_{M}}^{\mathbf{H}}\right|^{2}}{\left|\mathbf{w}_{L_{M}}^{\mathrm{H}} \mathbf{h}_{B L_{M}}\right|^{2}}, \frac{\tilde{\gamma}_{1}^{1} \tilde{\sigma}_{1,1}^{2}\left\|\mathbf{w}_{1}^{1 \mathrm{H}}\right\|^{2}}{\left|\mathbf{w}_{1}^{1 \mathrm{H}} \mathbf{h}_{F 1}^{1}\right|^{2}}, \ldots,\right.} \\
& \left.\frac{\tilde{\gamma}_{1}^{1} \tilde{\sigma}_{L_{F}, 1}^{2}\left\|\mathbf{w}_{L_{F}}^{1 \mathrm{H}}\right\|^{2}}{\left|\mathbf{w}_{L_{F}}^{1 \mathrm{H}} \tilde{\mathbf{h}}_{F L_{F}}^{1}\right|^{2}}, \ldots, \frac{\tilde{\gamma}_{1}^{K} \tilde{\sigma}_{1, K}^{2}\left\|\mathbf{w}_{1}^{K \mathrm{H}}\right\|^{2}}{\left|\mathbf{w}_{1}^{K \mathrm{H}} \tilde{\mathbf{h}}_{F 1}^{K}\right|^{2}}, \ldots, \frac{\tilde{\gamma}_{L_{F}}^{K} \tilde{\sigma}_{L_{F}, K}^{2}\left\|\mathbf{w}_{L_{F}}^{K \mathrm{H}}\right\|^{2}}{\left|\mathbf{w}_{L_{F}}^{K \mathrm{H}} \tilde{\mathbf{h}}_{F L_{F}}^{K}\right|^{2}}\right]^{\mathrm{T}} .
\end{aligned}
$$

Now, we want to find the beamforming vectors which maximize SINR as:

$$
\mathbf{w}_{\mathrm{SINR}}=\arg \max _{\mathbf{w}}\{S I N R\} .
$$

These optimum beamforming weights are computed by:

$$
\begin{aligned}
& \mathbf{W}_{l_{M}}=c_{l_{M}}\left(\sum_{\substack{i=1 \\
i \neq l_{M}}}^{L_{M}} \tilde{p}_{i} \mathbf{h}_{B i} \mathbf{h}_{B i}^{\mathrm{H}}+\sum_{k=1}^{K} \sum_{j=1}^{M_{F}} \tilde{p}_{j}^{k} \mathbf{h}_{B j}^{k} \mathbf{h}_{B j}^{k \mathrm{H}}+\sigma_{l_{M}}^{2} \mathbf{I}_{M_{M}}\right)^{-1} \mathbf{h}_{B l_{M}}, \\
& \mathbf{w}_{l_{F}}^{k}=c_{l_{F}}^{k}\left(\sum_{i=1}^{L_{M}} \tilde{p}_{i} \mathbf{h}_{F i}^{k} \mathbf{h}_{F i}^{k \mathrm{H}}+\sum_{\substack{j=1 \\
j \neq l_{F}}}^{L_{F}} \tilde{p}_{j}^{k} \tilde{\mathbf{h}}_{F j}^{k} \tilde{\mathbf{h}}_{F j}^{k \mathrm{H}}+\tilde{\sigma}_{j, k}^{2} \mathbf{I}_{M_{F}}\right)^{-1} \tilde{\mathbf{h}}_{F l_{F}}^{k}
\end{aligned}
$$

where $c_{l_{M}}$ and $c_{l_{F}}^{k}$ are chosen such that $\left\|\mathbf{w}_{l_{M}}\right\|^{2}=\left\|\mathbf{w}_{l_{F}}^{k}\right\|^{2}=1$. The optimization algorithm is summarized in Fig. 2.

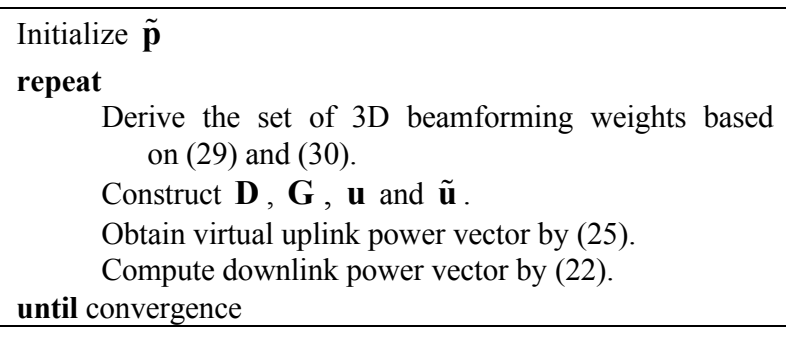

Fig. 2. Summarized form of the iterative algorithm for joint power control and 3D beamforming.

In order to show that the proposed algorithm converges to a feasible solution, assume that there is a set of weight vectors $\mathbf{w}$, for which $\rho\left(\mathbf{D G}^{\mathrm{T}}\right)<1$ where $\rho(\cdot)$ defines the spectral radius of a matrix. The matrix $\mathbf{I}-\mathbf{D G} \mathbf{G}^{\mathrm{T}}$ is then invertible and $\tilde{\mathbf{p}}=\left(\mathbf{I}-\mathbf{D G}^{\mathrm{T}}\right)^{-1} \tilde{\mathbf{u}}$ minimizes the objective function for a fixed set of weight vectors. For any feasible $\mathbf{w}$, the vector $\tilde{\mathbf{p}}$ can be computed as the limit of the iteration as:

$$
\tilde{\mathbf{p}}^{(n+1)}=\Psi^{w}\left(\tilde{\mathbf{p}}^{(n)}\right)
$$

where $\tilde{\mathbf{p}}^{(n)}$ represents the power vector at the $n$-th iteration and $\Psi^{w}$ is a beamforming dependent mapping which maps $\tilde{\mathbf{p}}^{(n)}$ to $\tilde{\mathbf{p}}^{(n+1)}$. Starting from any arbitrary initial power vector $\mathbf{p}^{0}$, the mapping $\Psi^{w}$ will converge to the optimal power vector $\tilde{\mathbf{p}}^{*}$, where $\tilde{\mathbf{p}}^{*}=\Psi^{w}\left(\tilde{\mathbf{p}}^{*}\right)$, i.e. $\tilde{\mathbf{p}}^{*}$ is the fixed point of the mapping $\Psi^{w}$. Furthermore, since the fixed point of this mapping is unique, the optimal weight vector $\mathbf{w}^{*}$ is also unique. Therefore, our proposed joint power control and $3 \mathrm{D}$ beamforming algorithm converge to a fixed power allocation.

As a result, the virtual uplink gain matrix $\mathbf{D G}^{\mathrm{T}}$ and consequently, the downlink gain matrix DG converge to constant matrices. The feasibility of the virtual uplink network means that the uplink gain matrix converges to a matrix whose eigenvalues are inside the unit circle. Since the eigenvalues of the uplink and downlink gain matrix are the same, the downlink gain matrix is an asymptotically constant matrix whose eigenvalues are inside the unit circle. Then, the downlink iteration is also convergent, and the algorithm converges to a feasible solution.

\section{Numerical Simulation Results}

In this section, we numerically evaluate our proposed joint power control and 3D beamforming scheme. Consider a cellular system with a radius of $150 \mathrm{~m}$ at $3.5 \mathrm{GHz}$. The $\mathrm{BS}$ is located in the center of the cell. There are 4 femtocells and each has 3 FUs. The BS and all FAPs are equipped with a planar array of printed dipole antennas. The height of the BS and the FAPs are $10 \mathrm{~m}$ and $2.5 \mathrm{~m}$, respectively, while the user's antenna height is $1.5 \mathrm{~m}$. The average power of the background noise is $-90 \mathrm{dBm}$. Initial transmission power of the BS and all FAPs to each user is $1 \mathrm{~mW}$ and the minimum required (target) SINR of all users is set to $20 \mathrm{~dB}$. 
First, we investigate convergence of the iterative proposed algorithm. The BS and each FAP have $8 \times 4$ and $2 \times 2$ antenna elements, respectively. There are 16 MUs in the microcellular area. Figures 3 and 4 illustrate the convergence behavior of the proposed scheme. It is observed that the proposed joint power control and 3D beamforming algorithm converges within a few iterations.

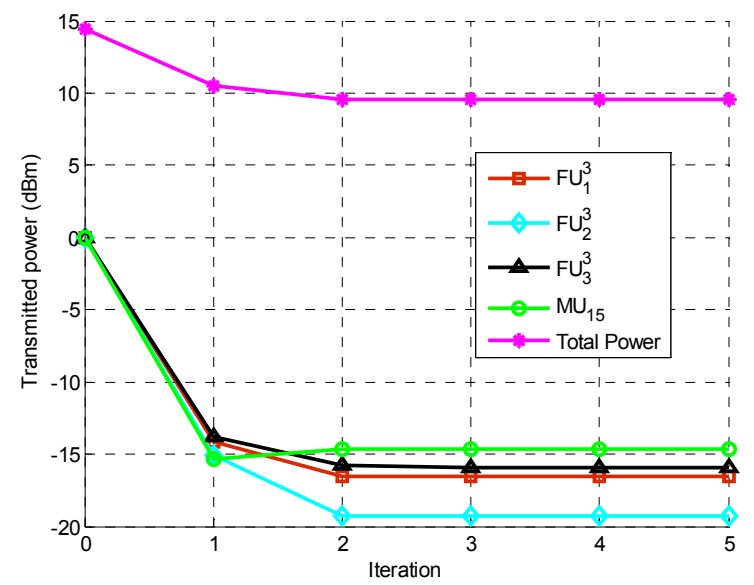

Fig. 3. Convergence of power $\left(L_{M}=16, L_{F}=3\right.$, BS: $8 \times 4$, FAPs $=2 \times 2$ and $\tilde{\gamma}=20 \mathrm{~dB}$ ).

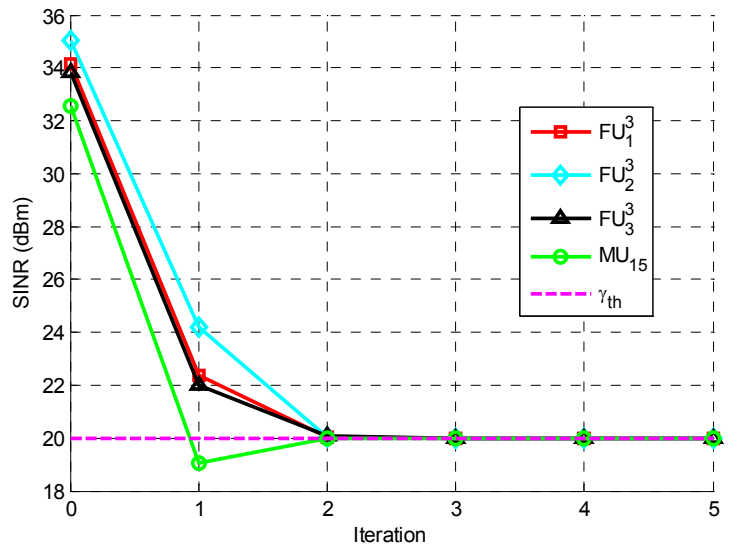

Fig. 4. Convergence of $\operatorname{SINR}\left(L_{M}=16, L_{F}=3\right.$, BS: $8 \times 4$, FAPs $=2 \times 2$ and $\tilde{\gamma}=20 \mathrm{~dB}$ ).

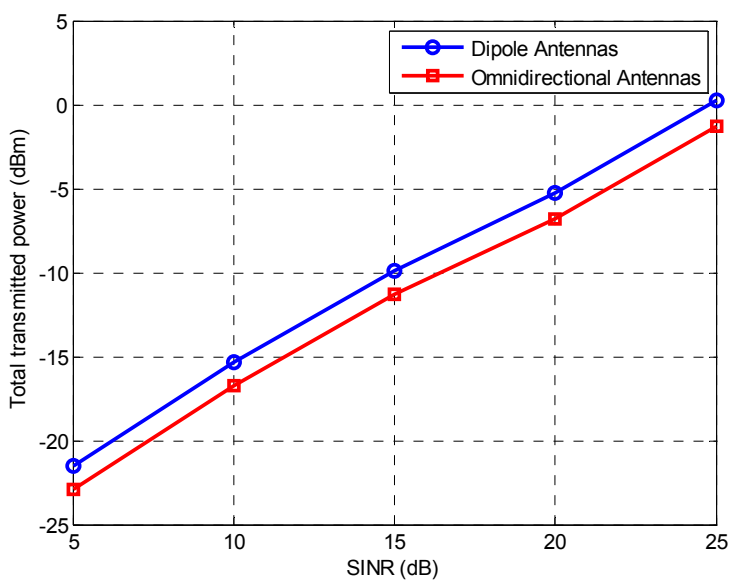

Fig. 5. Comparison of total transmitted power versus different target SINR values for omnidirectional and dipole array antennas $\left(L_{M}=4, L_{F}=3\right.$, BS: $4 \times 4$, FAP: $\left.2 \times 2\right)$.

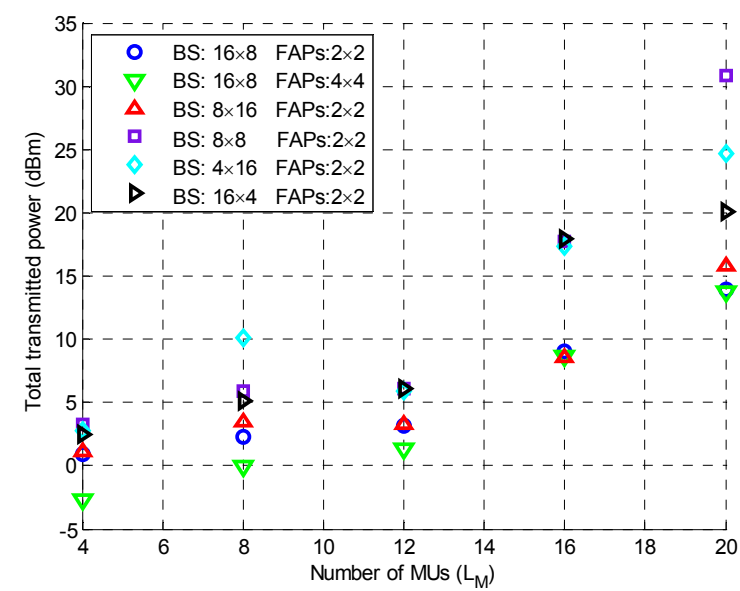

Fig. 6. Total transmitted power versus number of MUs $\left(L_{F}=3\right.$ and $\left.\tilde{\gamma}=20 \mathrm{~dB}\right)$

In order to show the effects of the antenna pattern on the system performance, we compare total transmitted power of the system where BS and the FAPs are equipped with arrays of printed dipole antennas to the case where all antennas are omnidirectional. The related simulation results are presented for different SINR values in Fig. 5, where the number of MUs is 8 and the BS and each FAP have $4 \times 4$ and $2 \times 2$ antenna elements, respectively. This figure shows that the system with printed dipole antennas requires about 1.5 times more power than a system equipped with omnidirectional array elements. This important result confirms that the exact antenna pattern should be considered during the system design phase.

Total transmitted power of the system versus the number of MUs for various BS and FAPs array configurations is presented in Fig. 6. It is seen that the power consumption of the system is increased with a greater number of MUs. However, by applying more antennas at the BS or FAPs, we can reduce total required power. Furthermore, different array configuration with the same number of antenna elements can change total transmitted power. For example, when the system serves 20 MUs, total transmitted power is $30.80 \mathrm{dBm}$ if the $\mathrm{BS}$ is equipped with 64 antennas arranged as an $8 \times 8$ array. The power is reduced to $13.88 \mathrm{dBm}$ for a scenario where the BS is equipped with 128 antennas configured as a $16 \times 8$ array. This results in more than $16.9 \mathrm{~dB}$ reduction in the power consumption of the whole system. However, with 64 antennas arranged as a $16 \times 8$ array, the power is also reduced to $13.74 \mathrm{dBm}$. This means that with the same number of BS antennas, the system can achieve about $17 \mathrm{~dB}$ reduction in the total transmitted power. Accordingly, precise attention should be paid for selection of the BS and FAPs array sizes and their configuration to minimize consumed power of the system.

\section{Conclusion}

In this paper, an iterative joint power control and 3D pattern-dependent beamforming algorithm is proposed to 
minimize total transmitted power of a femtocell system deployed in a macrocellular environment. This algorithm finds the 3D beamforming and power allocations of the FAPs and macrocell BS while satisfying QoS requirements of both macrocell and femtocell networks. In the proposed iterative algorithm, the exact radiation pattern of the BS and FAP antennas are used through the optimization process.

For performance evaluation of the proposed algorithm, a typical cellular area with a number of femtocells is considered. The simulations demonstrate that the algorithm converges within a few iterations. We investigate the effects of the antennas radiation pattern on the total system transmitted power. The simulations show that approximating array elements with omnidirectional antennas, performed in the previous literatures, can lead to underestimation of the total system consumed power. It is also observed that by appropriate configuration of the array elements, total transmitted power of the system can decrease considerably while the number of array elements is constant. The simulations confirm that the proposed technique can effectively use to reduce consumed power of the macrocell-femtocell heterogeneous networks.

\section{References}

[1] CHANDRASEKHAR, V., ANDREWS, J. G., GATHERER, A Femtocell networks: a survey. IEEE Communications Magazine, 2008, vol. 46, no. 9, p. 59-67. DOI: 10.1109/MCOM.2008.4623708

[2] CLAUSSEN, H., HO, L. T. W., SAMUEL, L. G. An overview of the femtocell concept. Bell Labs Technical Journal, 2008, vol. 13, no. 1 , p. 221-245. DOI: $10.1002 /$ bltj.20292

[3] ZHANG J., DE LA ROCHE, G. Femtocells: Technologies and Deployment. John Wiley \& Sons, 2010. ISBN: 978-0-470-74298-3

[4] ZAHIR, T., ARSHAD, K., NAKATA, A., MOESSNER, K. Interference management in femtocells. IEEE Communications Surveys and Tutorials, 2013, vol. 15, no. 1, p. 293-311. DOI: 10.1109/SURV.2012.020212.00101

[5] KAUFMAN, B. LILlEBERG, J. AAZHANG, B. Femtocell architectures with spectrum sharing for cellular radio networks. International Journal of Advances in Engineering Sciences and Applied Mathematics, 2013, vol. 5, no. 1, p. 66-75. DOI: $10.1007 / \mathrm{s} 12572-013-0083-5$

[6] DA COSTA, G. W. O. Dynamic Spectrum Sharing among Femtocells-Coping with Spectrum Scarcity in $4 G$ and Beyond. Ph.D Thesis. Aalborg University, Denmark, 2012. Available at: http://vbn.aau.dk/files/71879264/phdthesis_gustavowagner_final.pdf

[7] JIMING, C., PENG, W., JIE, Z. Adaptive soft frequency reuse scheme for in-building dense femtocell networks. In Proceedings of the IEEE International Conference on Communications in China (ICCC). Aug. 2012, p. 589-593. DOI: 10.1109/CC.2013.6457529

[8] ELEBI, Y., LI, H., DANESHMAND, M., WANG, C., ZHAO, W. Energy-efficient femtocell networks: Challenges and opportunities. IEEE Wireless Communications, 2013, vol. 20, no. 6, p. 99-105. DOI: 10.1109/MWC.2013.6704480

[9] MURAKAMI, T., KUDO, R., ISHIHARA, K., HONMA, N., MIZOGUCHI, M. Cooperative interference management by beam tilt and power controls in an indoor multi-cell environment. In
Proceedings of the 7th European Conference on Antennas and Propagation (EuCAP). Gothenburg (Sweden), Apr. 2013, p. 643 to 647. ISBN: 978-88-907018-3-2

[10] ZAHIR, T., ARSHAD, K., KO, Y., MOESSNER, K. A downlink power control scheme for interference avoidance in femtocells. In Proceedings of the 7th International Wireless Communications and Mobile Computing Conference (IWCMC). Istanbul (Turkey), July 2011, p. 1222-1226. DOI: 10.1109/IWCMC.2011.5982714

[11] OH, D.-C. LEE, H.-C. LEE, Y.-H. Power control and beamforming for femtocells in the presence of channel uncertainty. IEEE Transactions on Vehicular Technology, 2011, vol. 60, no. 6, p. 2545-2554. DOI: 10.1109/TVT.2011.2158615

[12] MO, R., QUEK, T. Q. S., HEATH JR., R. W. Robust beamforming and power control for two-tier femtocell networks. In Proceedings of the IEEE 73rd Vehicular Technology Conference (VTC Spring). May 2011, p. 1-5. DOI: 10.1109/VETECS.2011.5956628

[13] OH, D.-C., LEE, H.-C., LEE, Y.-H. Joint power control and spatial beamforming of cognitive radio based femtocell network with limited feedback. In Proceedings of the Korea Information and Communications Society. 2010, vol. 2, p. 69-70. Available at: www.dbpia.co.kr/Article/NODE02084470

[14] CHAN, A. M., LEE, I. A new reduced-complexity sphere decoder for multiple antenna systems. In Proceedings of the IEEE International Conference on Communications (ICC). Apr. 2002, p. 460-464. DOI: 10.1109/ICC.2002.996896

[15] SUNG, H., PARK, S. H., LEE, K. J., LEE, I. Linear precoder designs for $\mathrm{K}$-user interference channels. IEEE Transactions on Wireless Communications, 2010, vol. 9, no. 1, p. 291-301. DOI: 10.1109/TWC.2010.01.090221

[16] LEE, H., LEE, B., LEE, I. Iterative detection and decoding with an improved VBLAST for MIMO-OFDM systems. IEEE Journal on Selected Areas in Communications, 2006, vol. 24, no. 3, p. 504-513. DOI: 10.1109/JSAC.2005.862400

[17] ZHENG, W., SU, T., LI, W., LU, Z., WEN, X. Distributed energyefficient power optimization in two-tier femtocell networks. In Proceedings of the IEEE International Conference on Communications (ICC). Apr. 2011, p. 5767-5771. DOI: 10.1109/ICC.2012.6364882

[18] AN, C., XIE, R., JI, H. Pricing and power control for energyefficient radio resource management in cognitive femtocell networks. International Journal of Communication Systems, 2015, vol. 28 , no. 4 , p. $743-761$. DOI: $10.1002 /$ dac. 2700

[19] MAO, T., FENG, G., LIANG, L., QIN, S., WU, B. Distributed energy-efficient power control for macro-femto networks. IEEE Transactions on Vehicular Technology, 2016, vol. 65, no. 2, p. 718-731. DOI: 10.1109/TVT.2015.2402618

[20] LI, Y., JI, X., LIANG, D., LI, Y. Dynamic beamforming for threedimensional MIMO technique in LTE-advanced networks. International Journal of Antennas and Propagation, 2013, 8 p. DOI: $10.1155 / 2013 / 764507$

[21] RAZAVIZADEH, S. M., AHN, M., LEE, I. Three dimensional beamforming: New enabling technology for fifth generation wireless networks. IEEE Signal Processing Magazine, 2014, vol. 31, no. 6, p. 94-101. DOI: 10.1109/MSP.2014.2335236

[22] ZHANG, Z., TEH, K. C., LI, K. H. Study of three-dimensional beamforming strategies in cellular networks with clustered user distribution. IEEE Transactions on Vehicular Technology, 2016, vol. 65 , no. 12 , p. 10208-10213. DOI: 10.1109/TVT.2016.2543259

[23] KYOSTI, P., MEINILA, J., HENTILA, L., et al. WINNER II Channel Models, ver 1.1, 2007.

[24] RASHID-FARROKHI, F., LIU, K. J. R., TASSIULAS, L. Transmit beamforming and power control for cellular wireless systems. IEEE Journal on Selected Areas in Communications, 1998, vol. 16, no. 8, p. 1437-1450. DOI: 10.1109/49.730452 
[25] SCHUBERT, M., BOCHE, H. A unifying theory for uplink and downlink multi-user beamforming. In Proceedings of the International Zürich Seminar on Broadband Communication. Feb. 2002, p. 27.1-27.6. DOI: 10.1109/IZSBC.2002.991770

[26] SCHUBERT, M., BOCHE, H. Solution of the multiuser downlink beamforming problem with individual SINR constraints. IEEE Transactions on Vehicular Technology, 2004, vol. 53, no. 1, p. 18 to 28. DOI: $10.1109 /$ TVT.2003.819629

\section{About the Author ...}

Narges NOORI received B.Sc., M.Sc. and Ph.D. degrees (with honors) from the Iran University of Science and
Technology (IUST), Tehran, Iran, all in Electrical Engineering, in 1998, 2000 and 2006, respectively. From June 2004 to April 2005, she was with the RF/Microwave and Photonics Group, University of Waterloo, Waterloo, Ontario, Canada as a visiting scholar. In May 2005, she joined the Iran Telecommunication Research Center (ITRC), Tehran, Iran where she is now working as an associate professor. In 2008, she received the best researcher award from the Iran ministry of ICT. She has participated and managed several research projects in radio communication group of ITRC. Her research interests include wireless communications, radiowave propagation and channel modeling. 